\title{
Las Políticas Educativas de Equidad en Hon- duras: un análisis desde la perspectiva de género con una mirada en la situación de niñas y adolescentes
}

\section{The Educational Policies of Equity in Hondu- ras: an analysis from a gender perspective with a look at girls and adolescents present situation}

\author{
Nolvia Verónica López Recinos* \\ nolvial@upnfm.edu.hn \\ Lauren Gardela Maldonado* \\ Imaldonado@upnfm.edu.hn \\ Mirian Ondina Jiménez Durón* \\ mjimenez@upnfm.edu.hn
}

\section{Resumen}

En el presente ensayo, se realiza La Política Nacional de la Mujer, un análisis de las políticas de La Ley Fundamental de Educación equidad en la educación desde y El Currículo Nacional Básico. una perspectiva de género, Tomando como enfoque el ciclo documentándose, a través de una de políticas de Stephen Ball, para revisión bibliográfica de comparar el contexto de diferentes estudios escritos sobre influencia, el contexto de el tema, incluyendo, los producción del texto de la política instrumentos legales hondureños: con el contexto de la práctica.

* Docente del Departamento de Ciencias de la Educación, Universidad Pedagógica Nacional Francisco Morazán y Estudiante del Doctorado Latinoamericano en Políticas Públicas y Formación Docente, UPNFM.

Recibido 30 de agosto 2017 / Aceptado 25 de octubre 2017.

<Paradigma> - Revista de Investigación Educativa. Año 24. No. 38 
Evidenciándose, en el contexto de la práctica que la equidad de género plasmada teóricamente en los instrumentos legales hondureños no se aplica, debido, a que en las prácticas pedagógicas, se sigue perpetuando el sexismo, la discriminación y la exclusión hacia el sexo femenino, y que en el acceso y permanencia en el Sistema Educativo hondureño, se refleja un $19 \%$ de analfabetismo y una temprana deserción escolar, siendo las niñas, las adolescentes, pertenecientes a los pueblos originarios $y$ afrodescendientes los más afectados, igualmente, por la falta de una educación sexual integral, el país ocupa el segundo lugar en Latinoamérica con mayor tasa de fecundidad en adolescentes.

Por tanto, no basta solo con cumplir los lineamientos y exigencias de los Tratados y Convenios Internacionales para la inclusión de la equidad de género en la producción del texto de la política, sino que también, hay que reflexionar para tener una mirada crítica ante las desigualdades en los micro espacios, donde conviven, tanto profesores como estudiantes.

Palabras clave: equidad, equidad de género, políticas educativas, exclusión, ciclo de análisis de políticas, contextos.

\section{Abstract}

The following essay presents and analysis of the equity policies in education from a gender perspective. This analysis is based on a bibliographical review of studies related to the topic, among them the Honduran legal instruments: The National Policy of women, the Fundamental Law of Education and the National Curriculum of Education. The approach is based on the policy cycle of Stephen Ball as to compare the context of influence, the context of policy text production and the context of practice. There is evidence which indicates that gender equity stated in the Honduran legal documents is not applied in the classroom where sexism, discrimination, and exclusion prevail. 
Las Políticas Educativas de Equidad en Honduras: un análisis desde la perspectiva de género con una mirada en la situación de niñas y adolescentes

As a result, we should not be limited to the inclusion of guidelines, demands based on treaties, participation in conferences and international agreements so that equity policies in education can become a reality but, reflect on the present situation and have a critical perspective on the inequalities between the sexes in the educational scenarios where students and teachers meet.
To conclude, it can be said that $19 \%$ of Honduran population is illiterate and is also the dropout rate among school children, being girls, women and ethnic minorities that most affected. Honduras also has the second place in Latin America with the largest adolescent fertility rate reflecting the lack of sexual education in the educational system.

Keywords: equity, gender equity, educational policies, exclusion, cycle of policy analysis, contexts.

\section{Introducción}

En una sociedad desigual y excluyente como América Latina, la incorporación de la mujer en los espacios educativos, no deja de ser sexista, en los roles de género que se imponen, en las actividades diarias, poniendo en duda, su capacidad para aprender las áreas cognoscitivas como Lógica - Matemática o Ciencia y Tecnología. Para una mejor compresión y análisis de este tema, se tomó como base el ciclo de políticas propuesto por Stephen Ball (Avelar, 2016, p. 3) el cual incluye tres elementos que se deben tomar en consideración para la interpretación: el contexto de influencia, el contexto de producción del texto de la política y el contexto de la práctica.

Este ensayo, está estructurado en cuatro apartados: El primero, comprende el posicionamiento conceptual sobre equidad que significa "educar de acuerdo con las diferencias y necesidades individuales, sin que las condiciones económicas, demográficas, geográficas, étnicas o de género, supongan un impedimento al aprendizaje" (Organización 
de las Naciones Unidas para la Educación, la Ciencia y la Cultura UNESCO, 2002, p. 4). Seguidamente del concepto de equidad de género, que implica "la imparcialidad en el trato que reciben mujeres y hombres, de acuerdo con necesidades respectivas, ya sea con un trato igualitario o con uno diferenciado, pero que se considera equivalente en lo que se refiere a los derechos, los beneficios, las obligaciones y las posibilidades" ((UNESCO, P, 106).Y por último la diferencia entre equidad e igualdad, donde, esta visión igualitaria, entra en crisis a medida que las sociedades distribuyen sus riquezas de una forma desigual e injusta (López, 2005, p.65).

El segundo apartado de este ensayo considera el modelo de análisis: ciclo de políticas, donde se conceptualiza la política pública como "un conjunto de decisiones interrelacionadas que son adoptadas por un actor o grupos de actores políticos, que involucran la definición de metas y medios, para su logro en el marco de una situación particular" (Jenkins, 1978; Dunn, 1994; Parsons, 1995; Fischer, Miller \& Sydney, 2007; 995 en Espinoza 2009, p. 3). Además, se conceptualiza, la política educativa, que se refiere a un conjunto de variables y dimensiones complejas y diversas, que actúan en diversos niveles, comprendiendo los procesos estructurales, de los conflictos institucionales y desde la acción e ideología de los diferentes colectivos que participan de la sociedad desde intereses y acciones distintas (Rivas 2004, p. 5). Con referencia a esto, Ball, en sus planteamientos, resalta la importancia de mirar todos los factores y los contextos que influyen en la política educativa, y no solo concentrarse en un elemento específico. Por consiguiente, se debe entender e interpretar la realidad, en la que se producen las políticas educativas y la incorporación de la perspectiva de género en las mismas, no de una forma lineal, sino a través de un esquema cíclico (Avelar 2016, p.6).

El tercer apartado incluye el análisis de la inclusión de la perspectiva de género en los instrumentos educativos legales de Honduras, donde se describe el contexto de influencia que son los lineamientos y exigencias de los Tratados y Convenios Internacionales para la inclusión de la equidad de género en la producción del texto de la política, entre ellos El Currículo Nacional Básico, La Ley Fundamental de Educación y 
Las Políticas Educativas de Equidad en Honduras: un análisis desde la perspectiva de género con una mirada en la situación de niñas y adolescentes

La Política Nacional de la Mujer. También se describe el contexto de la práctica, que es el análisis de la situación real en la que se encuentran las niñas y adolescentes hondureñas, en lo que se refiere a la inclusión en las practicas pedagógicas dentro del salón de clases, el acceso, la permanencia y el derecho a tener una educación sexual integral.

Y en el último apartado se concluye, que el Estado hondureño ha seguido los lineamientos y exigencias internacionales de los Tratados, Conferencias y Convenciones, el cual ha sido el contexto de influencia para incluir la equidad de género en la producción del texto de la política educativa y que en el contexto de elaboración de texto de la política, se evidencia que el discurso político visibiliza la inclusión de la equidad de género en los instrumentos educativos legales y oficiales, los cuales son el CNB, La Ley Fundamental de Educación y La Política Nacional de la Mujer, pero en el contexto de la práctica, el cual está más relacionado con las vivencias o cotidianidades que se dan en los espacios educativos, se continúa perpetuando el sexismo en la educación, relacionado con los roles femeninos y masculinos y en el acceso y permanencia se sigue manifestando una temprana deserción escolar entre niñas y adolescentes, una elevada tasa de fecundidad adolescente, por falta de una adecuada educación sexual científica y obligatoria.

\section{Discusión Teórica}

\section{Equidad}

Definir el término de equidad no es solo conceptualizarlo sino, plantearlo bajo la mirada de la igualdad de oportunidades y criterios, tales como la justicia y la inclusión, lo cual implica, "educar de acuerdo con las diferencias y necesidades individuales, sin que las condiciones económicas, demográficas, geográficas, étnicas o de género, supongan un impedimento al aprendizaje" (Organización de las Naciones Unidas para la Educación, la Ciencia y la Cultura UNESCO, 2002, p. 4).En ese 
sentido, si se logra una distribución adecuada de los recursos, para que todas las escuelas, ya sea que pertenezcan al sector público o privado, puedan brindar un aprendizaje de alta calidad, que dé la seguridad que todos los sujetos que forman parte del sistema educativo tengan la oportunidad de un egreso con el desarrollo de las capacidades cognitivas, afectivas, sociales y laborales.

En relación con esto, Schmelkes, S. (2006) plantea:

Una educación bien distribuida, supone poder arribar a puntos de llegada comunes desde puntos de partida sumamente diversos, lo que implica necesariamente currículos flexibles y capacidad de adaptación curricular y metodológica, por parte de los docentes, a las características específicas de un alumnado social y culturalmente diverso (...) Equidad, como bien sabemos, no es dar lo mismo a todos (eso sería igualdad), sino dar a cada uno lo que necesita y más, a los que más lo necesitan (p. 50).

Por otra parte, para Juan Carlos Tedesco (2008) "el ideal de justicia social asume hoy un significado renovado, frente a la tendencia a la exclusión, la desigualdad y la fragmentación que muestra el capitalismo ligado a la pura lógica del mercado" (p. 80), también considera que es necesario distinguir entre demanda y necesidad, ya que satisfacer demandas, no es lo mismo que satisfacer necesidades. Debido a que la demanda está desigualmente distribuida, por lo que debemos de prestar mucha atención, al proceso en el cual una necesidad se transforma en una demanda expresada socialmente.

Es así como, se deben analizar los cambios en los procesos que hoy en día generan desigualdad y la construcción social que impera sobre nuevas formas de exclusión, los cuales rebaten el principio de equidad. Igualmente, se debería de crear un nivel básico de equidad que "...se refiere a un desarrollo cognitivo básico, producido en los primeros años de vida y vinculado a una sana estimulación afectiva, buena alimentación estimulación y condiciones sanitarias adecuadas" (Tedesco, 2008, p. 83), y además una cohesión social que implica “ expansión de la cobertura educativa, el mejoramiento de los resultados 
Las Políticas Educativas de Equidad en Honduras: un análisis desde la perspectiva de género con una mirada en la situación de niñas y adolescentes

de aprendizaje y las políticas de renovación curricular que permitan aprendizajes relevantes para el desempeño productivo y ciudadano" (Tedesco, 2008, p. 83)" para que los niños y niñas que provienen de familias con un capital cultural menos favorecido, tengan la oportunidad de nivelarse y lograr un proceso educativo exitoso.

\section{Equidad de género}

Incluir la equidad de género en las políticas educativas, es necesario, para evitar la discriminación y exclusión de las niñas, adolescentes y mujeres adultas, en los espacios educativos, por lo que la UNESCO define el concepto de la siguiente forma:

La imparcialidad en el trato que reciben mujeres y hombres, de acuerdo con necesidades respectivas, ya sea con un trato igualitario o con uno diferenciado, pero que se considera equivalente en lo que se refiere a los derechos, los beneficios, las obligaciones y las posibilidades. En el ámbito del desarrollo, un objetivo de equidad de género a menudo requiere incorporar medidas encaminadas a compensar las desventajas históricas y sociales que arrastran las mujeres (UNESCO, P. 106)

Por lo tanto, incluir la equidad de género, es determinante para de construir los estereotipos que se han entretejido, en la vida de las personas, sobre lo que significa ser hombre y ser mujer, igualmente para visibilizar la participación de las niñas y las adolescentes en aquellos espacios donde históricamente, han estado en desventaja, solo por el hecho de pertenecer al sexo femenino.

\section{¿Es lo mismo equidad e igualdad?}

La equidad y la igualdad son dos principios que pueden ir de la mano. Sin embargo, cuando las sociedades se van tornando cada vez más estratificadas, es muy difícil aplicar el principio de igualdad, por lo que, está visión igualitaria, entra en crisis a medida que las sociedades distribuyen sus riquezas de una forma desigual e injusta (López, 2005, p. 65). 
En ese sentido, si lo aplicamos al contexto educativo, una oferta educativa igualitaria para todos pasa inevitablemente a reproducir y reforzar situaciones de discriminación, debido a que no se puede tratar de la misma forma a las personas que tienen necesidades educativas de aprendizaje, necesidades educativas especiales y culturales, la cual deja de tener efectos integradores frente a semejantes desigualdades sociales (López, 2005, pp. 66-69). Por lo tanto, la igualdad y la equidad no son lo mismo, siempre la equidad tomará en cuenta las diferencias históricas y políticas de cada sociedad.

\section{Políticas públicas y su definición conceptual}

La educación en Latinoamérica, principalmente en el siglo XX, se ha trasformado conforme a las reformas que se han generado en la región; caracterizadas por un importante proceso de transformación que ha implicado tres elementos fundamentales: en primer lugar, el papel social asignado a la educación, visualizándose como una dimensión central del desarrollo económico y social de los países, en segundo, la importancia de la educación para garantizar la igualdad de oportunidades, un elemento importante, porque ha implicado la transformación de los sistemas educativos para lograr una educación de mayor calidad con equidad, y el tercero, referido al significado político-ético atribuido a la educación para contribuir a la paz, la democracia y el desarrollo, (UNESCO 1998, p. 6). Acciones, encaminadas hacia la consecución de objetivos y la calidad que tanto se anhela en la educación, así como también, mejorar las condiciones sociales de la población.

Estas transformaciones, han permitido la generación de políticas públicas tendientes a responder a las demandas de la población. Para una mejor comprensión de lo que es una política pública, cabe aclarar, que este no es un término que tenga la misma acepción en todos los países, por tanto, es imperativo diferenciar dos conceptos:

Politics (política), policies (políticas). El primero es entendido como las relaciones de poder, los procesos electorales, las confrontaciones entre organizaciones sociales con el gobierno. El 
Las Políticas Educativas de Equidad en Honduras: un análisis desde la perspectiva de género con una mirada en la situación de niñas y adolescentes

segundo tiene que ver más con las acciones, decisiones y omisiones por parte de los distintos actores involucrados en los asuntos públicos (Aguilar y Lima 2009, p. 2).

No obstante, estas acciones que incluye la política pública, no se deben entender como cualquier acción del Estado o del gobierno, sino que "consiste en ser un conjunto de acciones intencionales y causales, orientadas a la realización de un objetivo de interés y/o beneficio público, cuyos lineamientos de acción, agentes, instrumentos, procedimientos y recursos se reproducen en el tiempo de manera constante y coherente (...)" (Aguilar y Lima 2009, p. 3).

Este conjunto de acciones emanadas desde los gobiernos, son respuestas a las necesidades de una sociedad, que al final se traducen en resultados. En relación con esto Meny \& Thoenig (1992 p. 89), hacen referencia en términos generales a política pública como el "resultado de la actividad de una autoridad investida de poder público y de legitimidad gubernamental". Por su parte, Espinoza, en su análisis de los conceptos que plantean, señala que coinciden en que el concepto debe comprenderse como "un conjunto de decisiones interrelacionadas que son adoptadas por un actor o grupos de actores políticos, que involucran la definición de metas y medios, para su logro en el marco de una situación particular" (Jenkins, 1978; Dunn, 1994; Parsons, 1995; Fischer, Miller \& Sydney, 2007; 995 en Espinoza 2009, p. 3).

De ahí que las decisiones o actividades que se emanan desde el Estado y que se traducen en políticas, deben estar configuradas en el marco de la resolución de problemas sociales, que puedan permitir mejorar la sinergia entre los actores que participan en la política pública, en pro del bienestar de la población y orientada al logro de objetivos definidos y no a otros intereses.

\section{Las políticas educativas}

Las políticas educativas, son un conjunto de acciones que los sistemas educativos utilizan para organizar y dirigir la educación, por tanto, el 
análisis de las mismas es de vital importancia para el buen funcionamiento y direccionamiento de los sistemas educativos, que permitan lograr los objetivos propuestos. Efectivamente, hablar de política educativa, es un asunto que va más allá de visualizarla exclusivamente como un problema de toma de decisiones por parte de las ámbitos administrativos y de los propios políticos; es referirse a un conjunto de variables y dimensiones complejas y diversas, que actúan en diversos niveles, comprendiendo los procesos estructurales de los conflictos institucionales y desde la acción e ideología de los diferentes colectivos que participan de la sociedad desde intereses y acciones distintas (Rivas 2004, p. 5).

Para una mayor comprensión de las políticas educativas, es necesario realizar análisis, partiendo de un posicionamiento epistemológico, que vaya enfocado a la realidad contextual, en donde se aplican las mismas. Tello \& Mainardes (2012), advierten que es necesario "que los estudios sobre cuestiones epistemológicas en política educativa no deben 'copiar' los realizados en otros países, sino, comenzar a pensar la dimensión epistemológica de los estudios en política educativa en la realidad Latinoamericana" (pp. 8-9). Cabe agregar que se debe tomar también la realidad específica de cada país, de donde se analiza la aplicabilidad y el impacto de cada política educativa. Por tanto, para elaborar el ensayo de las Políticas Educativas de Equidad en Honduras: un análisis desde la perspectiva de género, con una mirada en la situación de niñas y adolescentes, se tomó como base, el ciclo de políticas que ha propuesto Stephen Ball (Beech y Meo 2016), por ser uno de los investigadores referentes sobre políticas educativas.

Honduras, ha generado diferentes políticas educativas encaminadas a la mejora y buen funcionamiento del Sistema Educativo. Una de esas políticas, es la incorporación de la equidad de género a través del II Plan de Igualdad y Equidad de Género de Honduras 2010-2022, en pro de coadyuvar al acceso, permanencia y egreso satisfactorio de las niñas y adolescentes para no seguir fomentado una educación diferenciada por sexo. En este sentido, "los estudios feministas han puesto de manifiesto el papel fundamental de la escuela, en el proceso 
Las Políticas Educativas de Equidad en Honduras: un análisis desde la perspectiva de género con una mirada en la situación de niñas y adolescentes

y tratamiento de las diferencias entre hombres y mujeres, ya que el género se construye a lo largo el proceso de socialización" (Ballarin 2004, p. 35).

Es evidente entonces, el rol preponderante que tiene la escuela, pues es uno de los principales contextos en donde se construyen los procesos de socialización. Con referencia a esto, Ball, en sus planteamientos, resalta la importancia de mirar todos los factores y los contextos que influyen en la política educativa, y no solo concentrarse en un elemento específico. Por consiguiente, se debe entender e interpretar la realidad en la que se producen las políticas educativas y la incorporación de la perspectiva de género en las mismas, no de una forma lineal, sino a través de un esquema cíclico (Avelar 2016, p.6).

Por ello, para comprender mejor las diferencias marcadas entre hombres y mujeres, así como la influencia en la sociedad, se debe partir del análisis del ciclo de políticas de Ball, el que se refiere al análisis de las políticas educativas, como una serie de fases o ciclos continuos relacionados entre sí, señalando que, para comprender estas fases, se debe:

Pensar acerca de las políticas, no como un documento o una cosa, sino como una entidad social que se mueve a través del espacio y cambia mientras se mueve y cambia a las cosas mientras se mueve, cambia los espacios a través de los cuáles se mueve. Es decir que, cambia cosas y también es cambiada, mientras se mueve (Avelar 2016, p. 6).

En síntesis, lo que se busca con el ciclo de las políticas, es "trazar un mapa" del proceso de las políticas públicas, que ilustre la forma en que deben considerarse las diversas etapas del ciclo de las políticas, como fases más fluidas e interactivas, y no como un conjunto de partes que no tienen relación entre ellas (Parsons 2008, p. 232).

Este movimiento constante y cambiante de las políticas, es el que se tiene que analizar en los diferentes contextos en los que se produce e impacta la política porque en la medida que se conocen los resultados,

<Paradigma> - Revista de Investigación Educativa. Año 24. No. 38 
se pueden hacer las mejoras a las mismas. Por ende, es importante analizar "la variedad de formas en que han sido y son contempladas por las políticas educativas las diferencias de género y cómo estas han contribuido y contribuyen a la mayor o menor discriminación de las mujeres" (Ballarin 2004, p. 35).

Por lo tanto, para la realización de este análisis de las políticas educativas, desde una perspectiva de género, se tomó como base, el enfoque planteado por Ball, el cual incluye tres contextos: el contexto de influencia, el contexto de producción del texto de política y el contexto de la práctica. Para una mejor comprensión de estos se señala que:

Los contextos de influencia que se refieren al ámbito donde se origina la política, así como a los actores y factores que inciden directa o indirectamente en su génesis; la producción de texto que alude a los procesos que se utilizan para producir la política a nivel de texto, los valores y conceptos relacionados a ella, así como la audiencia a la cual está dirigida, y la práctica que se asocia a la implementación de la política y a los efectos que esta puede tener en distintos niveles y actores (Espinoza 2009, p. 5).

Cabe agregar, que posteriormente Ball, añadió dos contextos más: el contexto de los resultados (efectos) y el contexto de la estrategia política; el primero hace referencia a temas de justicia, la igualdad y la libertad individual. La idea de que las políticas tienen efectos, en lugar de simplemente resultados, se consideran la más adecuadas. El segundo contexto implica la identificación de un conjunto de actividades sociales y políticas que serían necesarias, para manejar las desigualdades creadas o reproducidas por la política, a medida avanza la aplicabilidad de la misma (Mainardes 2006).

De los anteriores planteamientos se deduce que, en los contextos actuales se visualizan nuevos escenarios de influencia y de análisis de las políticas educativas, donde hay mayores niveles de participación 
Las Políticas Educativas de Equidad en Honduras: un análisis desde la perspectiva de género con una mirada en la situación de niñas y adolescentes

de los principales actores del proceso. Es evidente entonces que "la política educativa está siendo creada por nuevos actores que establecen nuevas fuentes de autoridad" (Beech y Meo 2016, p. 7).

Inclusión de la perspectiva de género en los instrumentos educativos legales de Honduras (Política Nacional de la Mujer, Ley Fundamental de Educación, Currículo Nacional Básico)

Instituto Nacional de la Mujer (INAM)

Para tratar el tema de la equidad, Honduras cuenta con una agenda política, para la igualdad y equidad de género, cuyo ente rector es $E I$ Instituto Nacional de la Mujer (INAM), delegándosele la función estratégica para que el Estado asuma la responsabilidad, frente a los compromisos internacionales y la normativa jurídica, para promover los derechos humanos de las mujeres (INAM, 2010).

En el eje cuatro, El INAM tiene seis políticas educativas que propone la equidad de género en el Sistema Educativo hondureño, de las cuales se analizan cuatro en este ensayo, utilizando para ello, el ciclo de políticas de Stephen Ball, específicamente el contexto de influencia, el contexto de producción del texto de política y el contexto de la práctica.

En ese sentido, la política 1 el eje cuatro del INAM, dice lo siguiente: El Estado promueve y garantiza una educación laica, no sexista y no discriminatoria, a través de la coevaluación, la incorporación de género en el currículo, el uso de metodologías y materiales educativos fundamentados en los Derechos Humanos, y la igualdad de género (INAM, 2010, p. 108).

Iniciando con el contexto de influencia de esta primera política de la mujer, la cual surge con la exigencia de los Tratados y Convenciones Internacionales que se han realizado, para que sea abolida la discriminación por razones de género. Al respecto, Honduras, como Estado miembro de La Organización de las Naciones Unidas (ONU) y 
de La Organización de los Estados Americanos (OEA), ha suscrito la mayoría de los tratados y convenciones. Por lo tanto, se ha inscrito en el Sistema Universal e Interamericano de protección de los Derechos Humanos, así mismo, en cuanto a los Tratados y Convenciones de los Derechos Humanos de las mujeres.

Honduras ratificó en 1983, la Convención sobre la eliminación de todas las formas de discriminación contra la mujer (CEDAW) y, en 1995, la Convención Interamericana para Prevenir, Sancionar y Erradicar la Violencia contra la Mujer, Igualmente, suscribió la plataforma de Acción de Beijing (1995), así como la Declaración de los Objetivos de Desarrollo del Milenio ODM (2000) (INAM, 2010, pp. 17-18). A partir de este contexto de influencia, el Estado de Honduras se ve en la obligatoriedad de cumplir con los acuerdos que ha firmado, lo que da el espacio para que el INAM, pueda incorporar el enfoque de género en el Currículo y el uso de metodologías y materiales educativos fundamentados en los Derechos Humanos y la igualdad de género (INAM, P. 108).

En este contexto de influencia descrito anteriormente se da paso al contexto de producción del texto de política, en el que se analiza el proceso de elaboración de los documentos legales que son textos oficiales, que tienen incorporado la perspectiva de género. Estos instrumentos son La Política Nacional de la Mujer, La Ley Fundamental de Educación y El Currículo Nacional Básico.

En el caso específico de La Política Nacional de la Mujer, el INAM compromete en su texto al Estado, para que promueva, a través del Currículo Nacional Básico (CNB), una educación laica, no sexista y no discriminatoria, mediante el uso de metodologías y materiales que reflejen la igualdad de género, lo cual se debería ver reflejado en el CNB. Por lo tanto, al revisar el CNB, se descubrió que este declara explícitamente "los textos serán elaborados y desarrollados desde una perspectiva de género que incluya entre otros: la eliminación de estereotipos por razones de sexo, promoción de responsabilidades domésticas compartidas [...] la utilización de un lenguaje incluyente 0 no-sexista" (CNB, 2003 p. 66). Igualmente, La Ley Fundamental de 
Las Políticas Educativas de Equidad en Honduras: un análisis desde la perspectiva de género con una mirada en la situación de niñas y adolescentes

Educación (2012), vela para que la educación "esté al alcance de todos los educandos sin discriminación y que debe ser laica" (p. 1 sección A).

Estos dos contextos de política, (CNB y Ley Fundamental de Educación) difieren mucho de lo que es el contexto de la práctica, ya que este último está más relacionado con las vivencias o cotidianidades que se dan en los espacios educativos locales. Si bien es cierto que los instrumentos legales incorporan en su texto el discurso con perspectiva de género, la realidad que se vive con las prácticas pedagógicas de los docentes dentro del aula de clases es otra.

En los centros educativos, se habla mucho de equidad, y sobre de la equidad de género. Sin embargo, no se reconoce cuando se está incluyendo la temática, debido a la poca socialización y formación de la misma, por lo que "La escuela tiene el encargo social de trasmitir los conocimientos, habilidades y valores culturales que son socialmente aceptados por una época determinada, sin embargo, todavía nos encontramos hoy que se continúan perpetuando estereotipos, que muchas veces se manifiestan de formas encubiertas" (Reinoso y Hernández, 2011, p.5). Coincidiendo lo anterior con el criterio de Alicia González y Beatriz Castellanos cuando expresaron que “... resulta necesario que el profesorado tenga conciencia de su papel en las transformaciones que pueden germinar desde la escuela misma, potenciando su capacidad para identificar los sesgos sexistas discriminatorios en la educación y las estrategias para luchar contra ellos" (en Reinoso y Hernández, 2011, p.5).

En consecuencia, en el contexto de la práctica, se observa que dentro del salón de clases los docentes clasifican las actividades por sexo; los estudiantes ya tienen asignado según su género, el rol que culturalmente, se considera legítimo, ya que "la escuela, reproduce estereotipos masculinos y femeninos, ya sea por acción o por omisión, que han sido construidos socioculturalmente, los que conducen a la desigualdad de género" (Reinoso y Hernández, 2011, p. 10). En el caso de la niña, se le asignan roles que están relacionados con la subjetividad, la cual, se traduce en subordinación y obediencia, 
mientras que a los niños se le asignan roles que están relacionados con la objetividad, la cual se traduce con la competitividad.

Una evidencia de los estereotipos que se ha naturalizado en las escuelas es el hecho de pensar que los niños son más diestros en actividades que involucren temas relacionados con Matemáticas, Tecnología, Álgebra, Física y Química; en tanto que a las niñas no se les entrena para estas áreas científicas, considerándose esto una acción que daña su autoestima e invisibilidad su inteligencia. Lo anterior se ve reflejado en los siguientes datos estadísticos:

"En 6 de cada 10 países, los chicos siguen teniendo mejores resultados en Matemáticas que las chicas, a las chicas, incluso a las mejores estudiantes les falta confianza en Matemáticas, 2 de cada tres chicas, frente a 1 de cada dos chicos, informan que a menudo se preocupan, porque temen que las clases de matemáticas les sean difíciles, Cuatro veces más chicos que chicas se plantean seguir una carrera profesional de ingeniería o informática" (OECD, PISA, 2012).

Al respecto Heller (1999) señala que:

"Generalmente, las niñas tienden a mostrar menos sus capacidades que sus compañeros varones. Así, las hipótesis explicativas que se manejan son las siguientes: diferencias de roles, valorando más a los chicos que a sus compañeras; menor formación de las niñas en tareas relacionadas con las matemáticas y las ciencias experimentales; menor competitividad y mejor trabajo colaborativo" (en Perales, 2012, p. 7).

En síntesis, estos estereotipos que se analizan en el contexto de la práctica son los que provocan más desigualdades de género, más inequidades y más violencia simbólica hacia las niñas y adolescentes, aumentando así la discriminación y exclusión en estos espacios.

En la política 2 del eje cuatro, se declara lo siguiente: "El Estado promueve la erradicación del analfabetismo de las mujeres, 
Las Políticas Educativas de Equidad en Honduras: un análisis desde la perspectiva de género con una mirada en la situación de niñas y adolescentes

considerando las realidades y necesidades específicas de las indígenas, afro descendientes y adultas mayores" (INAM, 2010, pp. 108). El contexto de influencia de esta política surge en el año 2000, donde la comunidad internacional asumió el compromiso de alcanzar las metas de La Educación para Todos y Los Objetivos de Desarrollo del Milenio. Por ende, el movimiento de La Educación para Todos es un compromiso mundial coordinado por la UNESCO que consiste en proporcionar educación básica de calidad a los niños, jóvenes y adultos, priorizando cinco objetivos en su agenda. Uno de ellos es el aumento de adultos alfabetizados.

Tal iniciativa comenzó en 1990, con La Conferencia Mundial sobre Educación para Todos, en Jomtien, Tailandia, haciendo hincapié en que la educación es un derecho humano. Por lo que, diez años más tarde, en El Foro Mundial sobre la Educación, en Dakar, Senegal, 164 gobiernos se comprometieron a hacer realidad La Educación Para Todos (UNESCO, 2011, p. 9). En ese sentido, el contexto de publicación del texto de la política del INAM, recomienda al Estado que promueva, en El Currículo Nacional Básico (CNB), la erradicación del analfabetismo de las mujeres, considerando las realidades de las mujeres indígenas, afro descendientes y adultas mayores.

En vista de ello, se lee explícitamente en el CNB (2013) lo siguiente: "Alfabetización funcional: para aquellas personas que no tuvieron acceso a la cultura escrita y al cálculo básico. Esta actividad se realiza con los esfuerzos del Estado y la sociedad civil, utilizando diferentes formas de entrega del servicio educativo" (p. 57). En cuanto a la producción del texto de La Ley Fundamental de Educación (2012), en el capítulo IV, de las modalidades de educación, en el artículo 27, hace referencia a la alfabetización, de la siguiente forma: "son las opciones organizativas y curriculares que ofrece el sistema nacional de educación, bajo los principios de equidad, integridad e inclusión de todos los grupos y personas, para dar respuestas a requerimientos específicos de formación, sean estos de carácter permanente o temporal" (p.7 sección A). 
Como bien se describe, los tres contextos de producción de texto de la política incluyen explícitamente el tema de equidad en la alfabetización, incluyendo la perspectiva de género, que son exigencia de las Convenciones y Tratados Internacionales. Cabe aclarar que Honduras, por ser parte de los países miembros, tiene que incluir dicho tema en su agenda política, no porque lo considere una necesidad, sino porque es una obligatoriedad.

Siguiendo con el análisis, llegamos al contexto de la práctica de esta política, y percibimos un contraste entre lo que dice el contexto del texto y lo que existe en la realidad de las mujeres. Sobre todo, a las que viven en el área rural y las pertenecientes a los pueblos indígenas y afro descendientes. De acuerdo a esto el PNUD, UNESCO y PREAL afirman que nuestro país se encuentra clasificado en el último tercio en cuanto al estado de la Educación Básica, con un índice de analfabetismo que alcanza el $19 \%$, el cual persiste por la temprana deserción escolar y también por la poca cobertura del sistema, por supuesto, estos datos se agravan, en el caso de los grupos más vulnerables, siendo las niñas, las mujeres, las minorías étnicas, la población rural y urbano marginal, las más afectadas (Agencia Española de Cooperación Internacional AECI, 2005, p. 27).

Por lo tanto, para el INAM (2010), la violación a los derechos de las mujeres indígenas y afro descendientes no está fundamentada únicamente en el género, sino también en su condición de clase y etnia. En algunos casos específicos de sociedades con estructuras socioculturales complejas, puede incluir la casta y la religión. Estos son los argumentos principales que explican su doble y triple discriminación. En este marco, las mujeres indígenas y afro descendientes señalan la necesidad de ampliar los Derechos Humanos, para que estos reconozcan y trabajen sobre la intersección de sus identidades individuales y colectivas, ya que, a pesar de haber emprendido múltiples esfuerzos en este sentido, todavía falta mucho por hacer en materia de políticas públicas (p. 101). Y es que, las niñas y las mujeres en sociedades patriarcales, suelen ser discriminadas, no solo por ser mujeres, sino que, si se es mujer y se pertenece a una 
Las Políticas Educativas de Equidad en Honduras: un análisis desde la perspectiva de género con una mirada en la situación de niñas y adolescentes

población indígena o afro descendiente, se le agrega un elemento más, que es la pobreza, entonces la niña, la adolescente y las mujeres adultas, reciben una triple discriminación y exclusión.

Por su parte la política 3 del eje cuatro, nos propone: "El Estado garantiza la igualdad entre hombres y mujeres en el acceso y permanencia en todo el Sistema Educativo" (INAM, 2010, pp. 109110). Los desafíos pendientes para que los niños y adolescentes, puedan ejercer el derecho a la educación, se encuentra comprendido en reconocidas iniciativas. El contexto de influencia está enmarcado, tanto en iniciativas internacionales como regionales. Dentro de las internacionales, surgen las cumbres de Educación para Todos de Jomtien y Dakar, así como, la identificación de Los Objetivos de Desarrollo del Milenio, en conjunto, con Las Metas Educativas para el año 2021 del bicentenario de la emancipación de América Latina. Dentro de las iniciativas nacionales, se han consolidado los esfuerzos, para concretar las metas de Educación para Todos (EFA, por su nombre en inglés). Recientemente el Gobierno Nacional de Honduras, planificó metas de mediano y largo plazo, que se evidencian en el Informe Nacional sobre la situación de inclusión y exclusión educativa, con el objetivo de avanzar hacia la identificación de los indicadores que articularán los esfuerzos de la sociedad, hacia la calidad y equidad educativa (República de Honduras. 2011).

De esta manera, en el contexto de producción del texto, la política del INAM, propone al Estado, garantizar el acceso y permanencia a la educación en igualdad de condiciones, para hombres y mujeres. La Ley Fundamental de Educación (República de Honduras, 2012), en sus artículos 2, 3, 8 y 9, establece la importancia del derecho a la educación, y específicamente, en su artículo 2, contempla el derecho que tiene toda persona de acceder al conocimiento, buscando maximizar el desarrollo de su personalidad y capacidades, en condiciones de libertad e igualdad, así como, el respeto a la dignidad que tiene como ser humano. 
De igual forma, plantea la necesidad de garantizar el acceso equitativo sin ninguna discriminación, mediante una educación integral de calidad, pública y obligatoria desde un (1) año de educación pre-básica, hasta el nivel medio, garantizado por mecanismos de cobertura ( $p .2$ sección A). En cuanto al CNB, dentro de sus objetivos específicamente en el inciso $h$, propone la atención de forma adecuada de la equidad social y de género, de manera que garantice, en igualdad de oportunidades, el desarrollo de sus potencialidades y habilidades (República de Honduras 2003, p. 16).

Como contexto de práctica, con base en los datos ofrecidos por La Secretaría de Educación de la República de Honduras del año 2011, se refleja que, si bien es cierto, se ha trabajado en garantizar el acceso a la educación, las cifras demuestran que en Prebásica ( 5 años) así como en el primero ciclo de Educación Básica (6, 7 y 8 años), existe aproximadamente 3 de cada 10 niños que aún no asisten a la escuela. Por tanto, la mitad de ellos permanecen fuera del ámbito educativo entre los 6 y los 7 años. Así mismo, para el término del año 2010, 126 mil estudiantes, entre 5 y 8 años, se encontraban fuera de la escuela, correspondiente a 56 mil niños de 5 años, 32 mil de 6 años, 25 mil de 7 años y 12 mil niños de 8 años (República de Honduras, 2011). Por lo que, en Honduras, la escolarización se extiende entre los 9 y los 11 años. En cambio, en la desescolarización se intensifica el abandono, convirtiéndose en un proceso que comienza a partir de los 12 años y se acentúa rápidamente en porcentajes donde, solo el $51,4 \%$ de los jóvenes de 15 años, está escolarizado, y de los de 16 años, el 48,7\% (p. 20).

Con relación, al género, vemos que el efecto del fracaso escolar y el abandono, van perfilando una estructura entre varones y mujeres, estando el nivel de preescolar, en una proporción similar de estudiante por sexo. No obstante, desde primer grado en adelante, este equilibrio comienza a perderse, siendo aún mayor el tránsito en los últimos años de Básica y Educación Media, donde se observa una significativa reducción de los inscritos (especialmente mujeres), existiendo una caída en la matrícula que pone en desventaja a las mujeres, con graves 
Las Políticas Educativas de Equidad en Honduras: un análisis desde la perspectiva de género con una mirada en la situación de niñas y adolescentes

efectos, a corto y mediano plazo, en cuestión educativa, así como lo veremos en el análisis de la política 5 (República de Honduras, 2011, p. 23).

La política 5 del eje cuatro plantea: "El Estado garantiza la obligatoriedad de la educación sexual, en el sistema educativo, desde una perspectiva de género y de Derechos Humanos, fundamentada en la promoción de la autoestima, procreación responsable y las relaciones familiares equitativas" (INAM, 2010, pp. 110-111). El contexto de influencia de esta política, también se enmarca, en los Convenios y Tratados Internacionales, de los cuales Honduras forma parte, en el plan de acción de La Conferencia Internacional de Población y Desarrollo del Cairo, donde los Estados de todo el mundo acordaron que "Ios derechos reproductivos abarcan ciertos derechos humanos que ya están reconocidos en las leyes nacionales, en los documentos internacionales sobre Derechos Humanos y en otros documentos pertinentes de las Naciones Unidas aprobados por consenso" y que "esos derechos se basan en el reconocimiento del derecho básico de todas las parejas e individuos a decidir libre y responsablemente el número de hijos, el espaciamiento de los nacimientos y el intervalo entre estos, y a disponer de la información y de los medios para ello y el derecho a alcanzar el nivel más elevado de salud sexual y reproductiva. También incluye su derecho a adoptar decisiones relativas a la reproducción sin sufrir discriminación, coacciones ni violencia, de conformidad con lo establecido en los documentos de Derechos Humanos" (Campaña por una Convención Interamericana de los Derechos Sexuales y los Derechos Reproductivos, 2010, p.2).

En el texto del CNB, el Estado involucra otros servicios para el fortalecimiento y desarrollo de la educación, como es "La Salud: higiene escolar, controles periódicos de la salud, alimentación escolar, salud sexual y reproductiva y seguro de salud para escolares" (CNB, 2003, P.18) Igualmente en La Ley Fundamental de Educación en el capítulo IV de las modalidades de educación, en el inciso 7, dice lo siguiente "La educación para la prevención y rehabilitación social está orientada 
[...] a niños y jóvenes en riesgo social, se proporciona como parte de programas integrales, con participación del gobierno central, local y la sociedad civil" (p. 7, sección A). Por su parte, La Secretaria de Salud, debido a los altos niveles de embarazos en adolescentes, se ha comprometido con la educación sexual y reproductiva del país, a través de La Estrategia Nacional para la Prevención del Embarazo en Adolescentes (ENAPREAH) que define las acciones comunitarias e institucionales que deben implementarse, para contribuir a disminuir este importante problema de salud (República de Honduras, 2012, p. 8).

En el contexto de la práctica, nos hallamos ante una situación crítica en Honduras, debido a que se ha elevado la tasa de embarazos en adolescentes, reportándose que, más de un $20 \%$ de las adolescentes ya son madres, mientras que, menos del $4 \%$ de los varones adolescentes son padres (También, Espinoza, 2015, p. 4).En ese sentido, la situación de los adolescentes en Honduras no escapa a la realidad de América Latina, los datos disponibles muestran la condición de vulnerabilidad en que se encuentra la población adolescente del país, teniendo una pubertad más temprana, el inicio más temprano de relaciones sexuales y el limitado acceso a la educación sexual, igualmente a los servicios de salud integral (ENAPREAH, p. 14).

Por la falta de programas acordes a la edad de postergar el inicio de su vida sexual o evitar el primer embarazo, Honduras se convierte en el segundo país de Latinoamérica con mayor fecundidad entre adolescentes, con una tasa de 102 por 1000 nacimientos. "Según la ENDESA para el año 2006, el 22\% de las mujeres hondureñas entre 15 y 19 años han estado alguna vez embarazadas. La peor parte la enfrentan las adolescentes pobres, adolescentes con bajos niveles educativos, y/o adolescentes originarias de los pueblos originarios y afro hondureños, cuyas estadísticas triplican a sus homólogas de los quintiles más ricos y con mejor educación" (En ENAPREAH, p.14).

Entre las estadísticas, que se hallan documentadas, no hay datos actualizados, sin embargo, los datos encontrados en el año 2012 muestran que el $17.4 \%$ de adolescentes en edades de 17 años ya son 
Las Políticas Educativas de Equidad en Honduras: un análisis desde la perspectiva de género con una mirada en la situación de niñas y adolescentes

madres de al menos un hijo y un $31.17 \%$ de adolescentes en edades de 18 años son madres de al menos un hijo. Sumado a lo anterior un $39.7 \%$ de las adolescentes entre 15 y 19 años que ya son madres, no tienen ningún grado de educación, un $34.2 \%$ tiene $1-3$ grados de primaria, un 24\% tiene 4-6 grados de primaria y un $8.1 \%$ tiene estudios de secundaria y un $0.0 \%$ no tiene estudios superiores (Mejía, 2012, p. 1)

\section{Conclusiones}

El Estado hondureño ha seguido los lineamientos y exigencias internacionales de los Tratados, Conferencias y Convenciones (CEDAW, IV Conferencia de Beijín, Conferencia de Jomtien, Convención de Belén do Pará, Convención del Cairo, OEA, entre otras) a través del Instituto Nacional de la Mujer, que es el ente rector. Y es a partir de este contexto de influencia cuando se incluye la equidad de género en los textos de política educativa al CNB y a La Ley Fundamental de Educación.

En el contexto de elaboración de texto de la política, se evidencia que el discurso político visibiliza la inclusión de la equidad de género en los instrumentos educativos legales y oficiales, los cuales son el CNB, La Ley Fundamental de Educación y la Política Nacional de la Mujer.

Sin embargo, en el contexto de la práctica, el cual está más relacionado con las vivencias o cotidianidades que se dan en los espacios educativos, se continúa perpetuando el sexismo en la educación, relacionado con los roles femeninos y masculinos, sobre todo en las actividades académicas en temas concernientes con las Matemáticas, Álgebra y Tecnología.

Igualmente, cuando se analiza el contexto de la práctica para promover la erradicación del analfabetismo en las mujeres, sobre todo las mujeres indígenas, afro descendientes y adultas mayores, se manifiesta que, según el PNUD, UNESCO Y PREAL, Honduras alcanza un $19 \%$ de analfabetismo, debido a una temprana deserción escolar, siendo las niñas, las mujeres y las minorías étnicas las más afectadas. En cuanto al análisis del contexto de la práctica, para tratar el tema 
del acceso y permanencia en el sistema educativo, se concluye que 3 de cada 10 niños no asisten a la escuela, siendo aún mayor el tránsito, en los últimos años de Básica y Educación Media, donde se observa una significativa reducción de los inscritos (especialmente mujeres), existiendo una caída en la matrícula que pone en desventaja a las mujeres, con graves efectos, a corto y mediano plazo.

Cuando se analiza el contexto de la práctica, de la obligatoriedad de la educación sexual, en el Sistema Educativo, desde una perspectiva de género y Derechos Humanos, la realidad es otra, ya que Honduras se convierte en el segundo país de Latinoamérica con mayor fecundidad entre adolescentes, con una tasa de 102 por cada mil nacimientos, donde las adolescentes con bajos niveles educativos, de estratos pobres, y las adolescentes de los pueblos originarios y afro descendientes son las más afectadas. 
Las Políticas Educativas de Equidad en Honduras: un análisis desde la perspectiva de género con una mirada en la situación de niñas y adolescentes

\section{Referencias Bibliográficas}

Agencia Española de Cooperación Internacional AECI (2005). Evaluación del programa de alfabetización de educación para adultos en Honduras y Nicaragua. Ministerio de Asuntos Exteriores y de Cooperación, 2005 Secretaría de Estado de Cooperación Internacional Dirección General de Planificación y Evaluación de políticas para el Desarrollo. Recuperado de: https://www.oecd.org/countries/honduras/ 46779335.pdf

Aguilar Astorga y Lima Facio (2009). ¿Qué son y para qué sirven las Políticas Públicas?, en Contribuciones a las Ciencias Sociales, septiembre 2009, www.eumed.net/rev/cccss/05/aalf.htm

Avelar, M. (2016). Entrevista con Stephen J. Ball: Su contribución al análisis de las políticas educativas (translated versión). Originally published as: Interview with Stephen J. Ball: analyzing his contribution to education policy research. Archivos Analíticos de Políticas Educativas, 24(24). Recuperado de: http://epaa.asu.edu/ojs/article/ viewFile/2368/1747

Ballarin, D. (2004). Géneroy políticas educativas. Revista de Educación, $\mathrm{N}^{\circ}$ 6: Universidad de Huelva. Recuperado de: http://rabida.uhu.es/ dspace/bitstream/handle/10272/1937/b15152844.pdf?seque

Beech, J. y Meo, A. I. (2016). Explorando el uso de las herramientas teóricas de Stephen J. Ball en el estudio de las políticas educativas en América Latina. Archivos Analíticos de Políticas Educativas, 24(23). http://dx.doi.org/10.14507/epaa.24.2417

Campaña por una Convención Interamericana de los Derechos Sexuales y los Derechos Reproductivos (mayo, 2010). Disponible en: https:// queeramnesty.ch/docs/propuesto2010_lima_esp.pdf 
Reinoso, I. y Hernández, J. (2011) La Perspectiva de Género en Educación. Revista académica semestral, volumen 3, No 28 Universidad de Málaga, Cuadernos de Educación y Desarrollo

Espinoza, I.; Espinoza, E; Sierra, S.; Sarmiento, L. \& Argueta, R. (2015). Proporción y características clínico - epidemiológicas de adolescentes embarazadas, en las zonas de influencia atendidas por médicos en servicio social, durante el período agosto 2015 - agosto 2016. Universidad Nacional Autónoma de Honduras, Facultad de Ciencias Médicas. Recuperado de: http://www.bvs.hn/Honduras/Embarazo/ Protocolo.Embarazo.en.Adolescentes.Corregida.09.Marzo.2016.pdf

Mejía, I. (2012) Adolescentes embarazadas en Honduras. Blog http:// embarazosadolescenteshn.blogspot.com/2012/08/estadisticasembarazo-y-maternidad-en.html

República de Honduras. Secretaría de Salud Estrategia Nacional para la Prevención del Embarazo en Adolescentes de Honduras (ENAPREAH) Tegucigalpa M.D. C. Septiembre 2012Disponible en: http:// www.paho.org/hon/index.php?option=com_docman\&view=download\& category_slug=salud-materna-nino-y-adolescente $\&$ alias $=332-$ estrategia-nacional-para-la-prevencion-del-embarazo en la adolescencia

Espinoza, O. (2009). Reflexiones sobre los conceptos de "política", políticas públicas y política educacional. Archivos Analíticos de Políticas Educativas, 17 (8). Recuperado de Fondo de las Naciones Unidas para la Infancia (UNICEF) (2010). Derechos de Niñas, Niños, Adolescentes y Mujeres, compendio legislativo internacional y nacional para la protección de sus derechos. Buenos aires: Argentina. Recuperado de https://www.unicef.org/argentina/spanish/Compedio_correg27_4.pdf

García, Perales, R.: "La educación desde la perspectiva de género", en ENSAYOS, Revista de la Facultad de Educación de Albacete, N. 27, 2012. Enlace web: http://revistas.uclm.es/index.php/ensayos. 
Las Políticas Educativas de Equidad en Honduras: un análisis desde la perspectiva de género con una mirada en la situación de niñas y adolescentes

Instituto Nacional de la Mujer INAM (2010). Política Nacional de la Mujer. II Plan de Equidad e Igualdad de Género de Honduras 20102022. Tegucigalpa, Honduras.

López, N. (2005) Equidad Educativa: Desafíos de la educación en el nuevo escenario latinoamericano. Instituto Internacional de Planeamiento de la Educación IIP-UNESCO, Sede Regional, Buenos Aires, Argentina.

Mainardes, J. (2006). Abordagem do ciclo de políticas: uma contribuição para a análise de políticas educacionais. Educ. Soc., Campinas, vol. 27, n. 94, p. 47-69. Recuperado de: http://www.scielo.br/pdf/es/v27n94/ a03v27n94.pdf

Meny, I. \& Thoenig. (1992). Las políticas públicas. (Versión española a cargo de Francisco Morata). Barcelona: Ariel, S. A.

Pallares F. (1988). Las políticas públicas: el sistema político en acción. Revista de Estudios Políticos (Nueva Época) N 62. Recuperado de https://dialnet.unirioja.es/descarga/articulo/27007.pdf

Parsons, W. (2007). Políticas Públicas: Una introducción a la teoría y la práctica del análisis de políticas públicas. FLACSO México. Buenos aires: Miño y Dávila.

Plan internacional y UNICEF. (2014). Vivencias y relatos sobre el embarazo en adolescentes: una aproximación a los factores culturales, sociales y emocionales a partir de un estudio en seis países de la región Panamá: Oficina Regional para Latinoamérica y el Caribe. Recuperado de: https://www.unicef.org/lac/UNICEF_PLAN_embarazo _adolescente_2015.pdf

Rebollo, M, García, R., Piedra, J., Vega, L. (2011). Diagnóstico de la cultura de género, actitudes del profesorado en la igualdad. Revista de Educación, 355. Mayo-agosto 2011, pp. 521-546 Fecha de entrada 25-11-2008 Fecha de aceptación: 10-03-2009

<Paradigma> - Revista de Investigación Educativa. Año 24. No. 38 
Reinoso, I. y Hernández, J. (2011) La Perspectiva de Género en Educación. Revista académica semestral, volumen 3, No 28. Cuadernos de Educación y Desarrollo Universidad de Málaga

República de Honduras. (2011). Informe nacional - la situación de inclusión y exclusión educativa. Asociación civil de educación para todos. Recuperado de: http://www.unicef.org/honduras/ Informe_nacional_situacion_inclusion_exclusion_educativa_honduras.pdf

República de Honduras. (2012). Ley Fundamental de Educación. ‥ ㅇ 32, 754. Honduras: La gaceta.

República de Honduras. Secretaría de Educación. (2003). Currículo Nacional Básico CNB. Tegucigalpa. Honduras.

República de Honduras. Secretaría de Salud. (2012). Estrategia Nacional para la Prevención del Embarazo en Adolescentes de Honduras (ENAPREAH) Tegucigalpa.

Rivas, Flores (2004). Política educativa y prácticas pedagógicas. Universidad de Málaga. BARBECHO, Revista de Reflexión Socioeducativa. Recuperado de: http://www.barbecho.uma.es/ DocumentosPDF/BARBECHO4/A7B4.pdf

Schmelkes, S. (2006). Diversidad, equidad, interculturalidad: las rupturas necesarias. p.50. Del texto, Calidad, Equidad y Reformas en la enseñanza. OEI, y Fundación Santillana. Madrid España.

Sistema de Naciones Unidas. (2010). Convención sobre la eliminación de todas las formas de discriminación contra la mujer (CEDAW), Protocolo Facultativo de la Convención sobre la eliminación de todas las formas de discriminación contra la mujer. Panamá. Recuperado de: https://www.unicef.org/panama/spanish/MujeresCo_web.pdf

Tedesco, J.C. (2008). Los temas de la agenda sobre gobierno y dirección de los sistemas educativos en América Latina. pp. 79-81. Del texto, 
Las Políticas Educativas de Equidad en Honduras: un análisis desde la perspectiva de género con una mirada en la situación de niñas y adolescentes

Calidad, Equidad y Reformas en la enseñanza. OEI, y Fundación Santillana. Madrid España.

Tello, C. y Mainardes, J. (2012). La posición epistemológica de los investigadores en Política Educativa: debates teóricos en torno a las perspectivas neo-marxista, pluralista y posestructuralista. Archivos Analíticos de Políticas Educativas, 20 (9). Recuperado http:// epaa.asu.edu/ojs/article/view/988

UNESCO (2002). En busca de la equidad en la educación secundaria: Encuentro regional sobre Educación para Todos en América Latina y el Caribe. Santiago de Chile. p. 4 Recuperado de: http://unesdoc.unesco. org/images/0016/001611/161191S.pdf

UNESCO (2011). "Toda persona tiene derecho a la educación". Publicado en noviembre de 2011 por la Organización de las Naciones Unidas para la Educación, la Ciencia y la Cultura 7, place de Fontenoy, 75352 París 07 SP, Francia.

UNESCO (2014). Indicadores UNESCO de cultura para el desarrollo, manual metodológico. Recuperado de http://es.unesco.org/creativity/ sites/creativity/files/digital-library/cdis/Iguldad\%20de\%20genero.pdf

UNESCO. (1998). Proyecto Principal de Educación en América Latina y el Caribe. Oficina Regional de Educación para América Latina y el Caribe. Boletín 45. Santiago, Chile. Disponible en: http:// unesdoc.unesco.org/images/0011/001131/113160s.pdf

<Paradigma> - Revista de Investigación Educativa. Año 24. No. 38 